\title{
52.
}

\section{SUR QUELQUES PROPRIÉTÉS DES DÉTERMINANTS GAUCHES.}

[From the Journal für die reine und angewandte Mathematik (Crelle), tom. XxxII. (1846), pp. 119-123.]

I.

JE donne le nom de déterminant gauche, à un déterminant formé par un système de quantités $\lambda_{r . s}$ qui satisfont aux conditions

$$
\lambda_{r .8}=-\lambda_{s . r}[r \neq s]
$$

J'appelle aussi un tel système, système gauche. On obtiendra des formules plus simples (quoique cette supposition ne soit pas essentielle), en considérant seu'ement les systèmes pour lesquels on a aussi

$$
\lambda_{r . r}=1
$$

Je suppose dans tout ce qui va suivre, que le déterminant est de l'ordre $n$, et que par conséquent les suffixes variables $r, s$, \&c., s'étendent toujours depuis l'unité jusqu'à $n$.

En posant les équations

$$
\Sigma_{r} \lambda_{r . s} x_{r}=P_{s}, \quad \Sigma_{s} \lambda_{r . s} x_{s}=Q_{r}
$$

j'exprime les systèmes inverses qui déterminent les $P, Q$ par les $x$, de la manière suivante:

$$
K x_{r}=\Sigma_{s} \Lambda_{r . s} P_{s} \text {, et } K x_{s}=\Sigma_{r} \Lambda_{r . s} Q_{r}
$$

où $K$ désigne le déterminant formé avec les quantités $\lambda_{r . s}$, et $\Lambda_{r . s}$ le coefficient différentiel de $K$ par rapport à $\lambda_{r . s}$; bien entendu, que la différentiation doit être 
effectuée avant d'avoir particularisé ces quantités par les équations (1), (2). On sait que ces fonctions $\Lambda$ satisfont aux conditions

$$
\left.\begin{array}{l}
\Sigma_{r} \lambda_{r .8} \Lambda_{r . s^{\prime}}=0, s \neq s^{\prime}, \\
\Sigma_{r} \lambda_{r .8} \Lambda_{r .8}=K, \\
\Sigma_{r} \lambda_{r .8} \Lambda_{r^{\prime} .8}=0, r \neq r^{\prime} \\
\Sigma_{s} \lambda_{r .8} \Lambda_{r .8}=K .
\end{array}\right\}
$$

Je tire des équations (4), en échangeant $r$ et $s$ dans la dernière de ces équations:

$$
\Sigma_{s} \Lambda_{r .8} P_{s}=\Sigma_{s} \Lambda_{s . r} Q_{s}
$$

et de là, en multipliant respectivement les différentes équations de ce système par $\lambda_{8^{\prime} . r}$, et prenant la somme de ces produits :

$$
\Sigma_{s}\left(\Sigma_{r} \lambda_{g^{\prime}, r} \Lambda_{r .8}\right) P_{s}=\Sigma_{s}\left(\Sigma_{r} \lambda_{s^{\prime} . r} \Lambda_{s, r}\right) Q_{s}
$$

On a d'abord par les équations (5)

$$
\Sigma_{s}\left(\Sigma_{r} \lambda_{s^{\prime} . r} \Lambda_{s, r}\right) Q_{s}=K Q_{\delta^{\prime}}
$$

puis par les équations (1) et (2)

$$
\Sigma_{r} \lambda_{8^{\prime} . r} \Lambda_{r . s}=2 \Lambda_{8^{\prime} . s}-\Sigma_{r} \lambda_{r .8^{\prime}} \Lambda_{r}
$$

c'est-à-dire par les équations (5):

et

$$
\left.\begin{array}{l}
\Sigma_{r} \lambda_{\delta^{\prime} . r} \Lambda_{r . s}=2 \Lambda_{8^{\prime} .8} ; \quad s^{\prime} \neq s \\
\Sigma_{r} \lambda_{\delta^{\prime} . r} \Lambda_{r . g^{\prime}}=2 \Lambda_{8^{\prime} .8^{\prime}}-K
\end{array}\right\}
$$

ce qui donne

$$
\Sigma_{s}\left(\Sigma_{r} \lambda_{\delta^{\prime} . r} \Lambda_{r .8}\right) P_{s}=2\left(\Sigma_{8} \Lambda_{\delta^{\prime} .8} P_{8}\right)-K P_{\delta^{\prime}}
$$

Substituant les équations (8) et (11) dans la formule (7), on obtiendra, en écrivant $r$ au lieu de $s^{\prime}$ :

$$
K Q_{r}=2\left(\Sigma_{s} \Lambda_{r . s} P_{s}\right)-K P_{r}
$$

et également

$$
K P_{s}=2\left(\Sigma_{r} \Lambda_{r . s} Q_{r}\right)-K Q_{s}
$$

Posant maintenant

$$
\left.\begin{array}{l}
K \alpha_{r .8}=2 \Lambda_{r .8} ; \quad r \neq s \\
K \alpha_{r . r}=2 \Lambda_{r . r}-K
\end{array}\right\}
$$

les formules (12), (13) se changeront en

$$
Q_{r}=\Sigma_{s} \alpha_{r . s} P_{s} \text { et } P_{s}=\Sigma_{r} \alpha_{r . s} Q_{r}
$$


équations qui sont nécessairement équivalentes. On a donc identiquement

(1) $\left\{\begin{array}{l}\Sigma_{r} \alpha_{r . s} \alpha_{r . s^{\prime}}=0 ; s \neq s^{\prime}, \\ \Sigma_{r} \alpha_{r . s} \alpha_{r .8}=1,\end{array}\right.$
(2) $\left\{\begin{array}{l}\Sigma_{s} \alpha_{r . s} \alpha_{r^{\prime} .8}=0 ; r \neq r^{\prime}, \\ \Sigma_{s s} \alpha_{r .8} \alpha_{r .8}=1:\end{array}\right.$

c'est-à-dire, on a trouvé un système de $n^{2}$ quantités $\alpha_{r, 8}$, fonctions explicites et rationnelles d'un nombre $\frac{1}{2} n(n-1)$ de variables indépendantes, qui satisfont identiquement aux formules $(16,1)$ et $(16,2)$. On sait qu'en géométrie cela veut dire que pour $n=2$ ou $n=3$ de tels systèmes donnent les coefficients propres à effectuer la transformation de deux systèmes de coordonnées rectangulaires; nous dirons par analogie, que des systèmes qui satisfont aux équations (16) pour une valeur quelconque de $n$, sont propres à effectuer la transformation entre deux systèmes de coordonnées rectangulaires. On a donc le théorème suivant:

Les coefficients propres à la transformation de coordonnées rectangulaires, peuvent être exprimés rationnellement au moyen de quantités arbitraires $\lambda_{r, s}$, soumises aux conditions

$$
\lambda_{s . r}=-\lambda_{s . r}[r \neq s] ; \lambda_{r . r}=1 .
$$

Pour développer les formules, il faut d'abord former le déterminant $K$ de ce système, puis le système inverse $\Lambda_{r, s}, \ldots$ et écrire

$$
K \alpha_{r . s}=2 \Lambda_{r . s}[r \neq s] ; \quad K \alpha_{r . r}=2 \Lambda_{r . r}-K
$$

ce qui donne le système cherché.

Soit par exemple $n=3$. Écrivons pour le système des quantités $\lambda_{r_{. s}}$ :

$$
\left.\begin{array}{rrr}
1, & \nu, & -\mu, \\
-\nu, & 1, & \lambda, \\
\mu, & -\lambda, & 1
\end{array}\right\}
$$

ce qui donne $K=1+\lambda^{2}+\mu^{2}+\nu^{2}$, et pour le système des fonctions $\Lambda_{r . s}$

$$
\left.\begin{array}{rrr}
1+\lambda^{2}, & \lambda \mu+\nu, & \nu \lambda-\mu, \\
\lambda \mu-\nu, & 1+\mu^{2}, & \mu \nu+\lambda, \\
\nu \lambda+\mu, & \mu \nu-\lambda, & 1+\nu^{2} .
\end{array}\right\}
$$

De là on obtient pour le système de coefficients $\alpha, \beta, \gamma ; \alpha^{\prime}, \beta^{\prime}, \gamma^{\prime} ; \alpha^{\prime \prime}, \beta^{\prime \prime}, \gamma^{\prime \prime}$ :

$$
\begin{aligned}
& K \alpha=1+\lambda^{2}-\mu^{2}-\nu^{2}, \quad K \alpha^{\prime}=2(\lambda \mu+\nu) \quad, \quad K \alpha^{\prime \prime}=2(\nu \lambda-\mu) \\
& K \beta=2(\lambda \mu-\nu) \quad, \quad K \beta^{\prime}=\left(1+\mu^{2}-\nu^{2}-\lambda^{2}\right), \quad K \beta^{\prime \prime}=2(\mu \nu+\lambda) \\
& K \gamma=2(\nu \lambda+\mu) \quad, K \gamma^{\prime}=2(\mu \nu-\lambda) \quad, \quad K \gamma^{\prime \prime}=\left(1+\nu^{2}-\lambda^{2}-\mu^{2}\right) ;
\end{aligned}
$$

ce qui se rapporte à la transformation

$$
\left.\begin{array}{ll}
x=\alpha x_{1}+\beta y_{1}+\gamma z_{1}, & x_{1}=\alpha x+\alpha^{\prime} y+\alpha^{\prime \prime} z, \\
y=\alpha^{\prime} x_{1}+\beta^{\prime} y_{1}+\gamma^{\prime} z_{1}, & y_{1}=\beta x+\beta^{\prime} y+\beta^{\prime \prime} z \\
z=\alpha^{\prime \prime} x_{1}+\beta^{\prime \prime} y_{1}+\gamma^{\prime \prime} z_{1}, & z_{1}=\gamma x+\gamma^{\prime} y+\gamma^{\prime \prime} z
\end{array}\right\}
$$


de deux systèmes de coordonnées rectangulaires. En effet, les coefficients $\lambda, \mu, \nu$ ont une signification géométrique: Les axes $A x_{1}, A y_{1}, A z_{1}$ vont coïncider avec les axes $A x, A y, A z$, par la rotation $\theta$ autour d'un certain axe $A R$ (qu'on peut nommer "Axe résultant"). En prenant $f, g, h$ pour les inclinaisons de cet axe à $A x, A y, A z$, on a $\lambda=\operatorname{tang} \frac{1}{2} \theta \cos f, \mu=\operatorname{tang} \frac{1}{2} \theta \cos g, \nu=\operatorname{tang} \frac{1}{2} \theta \cos h$. Cette expression de l'axe $A R$ est due à Euler; les quantités $\lambda, \mu, \nu$ ont été introduites pour la première fois, par M. Olinde Rodrigues, dans un mémoire "Sur les lois géométriques qui régissent les déplacements d'un système solide" (Liouville, tom. v. [1840]), où il donne [des expressions semblables à celles] qu'on vient de trouver ici, pour les coefficients de la transformation, en termes de $\lambda, \mu, \nu$. Ces mêmes quantités $\lambda, \mu, \nu$ (il $\mathrm{y}$ a à remarquer cela en passant) sont liées de la manière la plus étroite avec celles de la belle théorie de Sir W. Hamilton sur les Quaternions. Je les ai appliquées à la théorie de la rotation d'un corps solide. Avant de donner une idée des résultats auxquels je suis parvenu, je passe aux formules de transformation qui se rapportent au cas de $n=4$. Je prends ici pour le système des quantités $\lambda$ :

$$
\left.\begin{array}{rrrr}
1, & a, & b, & c, \\
-a, & 1, & -h, & g \\
-b, & h, & 1, & -f, \\
-c, & -g, & f, & 1,
\end{array}\right\}
$$

ce qui donne, en mettant pour abréger, $a f+b g+c h=\theta$,

$$
K=1+a^{2}+b^{2}+c^{2}+f^{2}+g^{2}+h^{2}+\theta^{2}
$$

et puis pour les quantités $\Lambda_{r .8}$ le système

$$
\left.\begin{array}{rrrr}
1+f^{2}+g^{2}+h^{2}, & f \theta+a+b h-c g, & g \theta+b+c f-a h, & h \theta+c+a g-b f, \\
-f \theta-a+b h-c g, & 1+f^{2}+b^{2}+c^{2}, & -c \theta-h+f g-a b, & b \theta+g+h f-c a \\
-g \theta-b+c f-a h, & c \theta+h+f g-a b, & 1+g^{2}+c^{2}+a^{2}, & -a \theta-f+g h-b c \\
-h \theta-c+a g-b f, & -b \theta-g+h f-c a, & a \theta+f+g h-b c, & 1+h^{2}+a^{2}+b^{2},
\end{array}\right\}
$$

de manière que pour

$$
\left.\begin{array}{llll}
K \alpha, & K \alpha^{\prime}, & K \alpha^{\prime \prime}, & K \alpha^{\prime \prime \prime}, \\
K \beta, & K \beta^{\prime}, & K \beta^{\prime \prime}, & K \beta^{\prime \prime \prime}, \\
K \gamma, & K \gamma^{\prime}, & K \gamma^{\prime \prime}, & K \gamma^{\prime \prime \prime}, \\
K \delta, & K \delta^{\prime}, & K \delta^{\prime \prime}, & K \delta^{\prime \prime \prime},
\end{array}\right\}
$$

on obtient le système suivant :

$$
\begin{aligned}
& f^{2}+g^{2}+h^{2}-a^{2}-b^{2}-c^{2}, \quad 2(f \theta+a+b h-c g) \\
& 2(g \theta+b+c f-a h) \quad, 2(h \theta+c+a g-b f) \\
& f \theta-a+b h-c g) \\
& \text {, } 1+f^{2}+b^{2}+c^{2}-g^{2}-h^{2}-a^{2} \text {, } \\
& 2(-c \theta-h+f g-a b) \quad, 2(b \theta+g+h f-c a) \\
& 9 \theta-b+c f-a h) \\
& 2(c \theta+h+f g-a b) \\
& 1+g^{2}+c^{2}+a^{2}-f^{2}-h^{2}-b^{2}, \quad 2(-a \theta-f+g h-b c) \\
& h \theta-c+a g-b f) \\
& 2(-b \theta-g+h f-c a) \\
& 2(a \theta+f+g h-b c) \\
& 1+h^{2}+a^{2}+b^{2}-f^{2}-g^{2}-c^{2},
\end{aligned}
$$

insi de suite pour des valeurs quelconques de $n$. 
II.

Maintenant je vais citer les formules que j'ai présentées dans le Journal de Cambridge, t. III. (1843) p. 225, [6], pour la rotation d'un corps solide autour d'un point fixe. Mettant, comme à l'ordinaire, les vitesses angulaires autour des axes principaux $p, q, r$, les moments du corps pour ces mêmes axes $=A, B, C$, et la fonction des forces $=V$ : les équations citées pourront être écrites sous la forme

$$
\begin{aligned}
& \qquad d=\frac{d p}{P}=\frac{d q}{Q}=\frac{d r}{R}=\frac{d \lambda}{\Lambda}=\frac{d \mu}{\mathrm{M}}=\frac{d \nu}{\mathrm{N}} \ldots \ldots \ldots \ldots \ldots \ldots \ldots \ldots \\
& P=\frac{1}{A}\left((B-C) q r+\frac{1}{2}\left\{\left(1+\lambda^{2}\right) \frac{d V}{d \lambda}+(\lambda \mu+\nu) \frac{d V}{d \mu}+(\lambda \nu-\mu) \frac{d V}{d \nu}\right\}\right) \\
& Q=\frac{1}{B}\left((C-A) r p+\frac{1}{2}\left\{(\mu \lambda-\nu) \frac{d V}{d \lambda}+\left(1+\mu^{2}\right) \frac{d V}{d \mu}+(\mu \nu+\lambda) \frac{d V}{d \nu}\right\}\right) \\
& R=\frac{1}{C}\left((A-B) p q+\frac{1}{2}\left\{(\nu \lambda+\mu) \frac{d V}{d \lambda}+(\mu \nu-\lambda) \frac{d V}{d \mu}+\left(1+\nu^{2}\right) \frac{d V}{d \nu}\right\}\right) \\
& \Lambda=\frac{1}{2}\left\{\left(1+\lambda^{2}\right) p+(\lambda \mu-\nu) q+(\lambda \nu+\mu) r\right\} \\
& \mathrm{M}=\frac{1}{2}\left\{(\mu \lambda+\nu) p+\left(1+\mu^{2}\right) q+(\mu \nu-\lambda) r\right\} \\
& \mathrm{N}=\frac{1}{2}\left\{(\nu \lambda-\mu) p+(\nu \mu+\lambda) q+\left(1+\nu^{2}\right) r\right\} .
\end{aligned}
$$

En effet, pour obtenir ces formules, il n'y a qu'à chercher au moyen de $\lambda, \mu, \nu$, et de leurs dérivées par rapport aux temps $\lambda^{\prime}, \mu^{\prime}, \nu^{\prime}$, l'expression de la fonction $T=\frac{1}{2}\left(A p^{2}+B q^{2}+C r^{2}\right)$ \{qui exprime la demi-somme des forces vives\}: cela fait, les formules générales que Lagrange a données pour la solution des problèmes de dynamique, conduisent immédiatement aux équations en question. Dans le mémoire cité j’ai intégré ces équations pour le cas où la fonction $V$ est zéro, et en prenant, comme dans la théorie ordinaire, le plan invariable pour plan des deux axes. Ce n'est que dernièrement que j'ai trouvé la manière convenable de traiter ce système d'équations; je le fais au moyen de deux nouvelles variables $\Omega, v$, entre lesquelles je trouve une équation différentielle dont les variables sont séparées, et j'exprime en termes de celles-ci les autres variables du problème, $\mathrm{y}$ compris le temps; et cela sans aucune supposition particulière, relative à la position des axes des coordonnées par rapport au plan invariable. Le développement de cette théorie paraîtra dans le prochain No. du Cambridge and Dublin Mathematical Journal, [37]. Je m'occupe aussi de la recherche des formules pour les variations des constantes arbitraires relatives aux perturbatrices. Il serait bien intéressant (comme problème d'analyse pure) d'étendre ces recherches au cas d'une valeur quelconque de $n$; il faudrait pour cela, chercher les valeurs des quantités analogues à $p, q, r$, former une fonction $T$, en prenant la somme des carrés de chacune de ces quantités, chaque carré multiplié par un coefficient constant, et puis former les équations $\frac{d}{d t} \cdot \frac{d T}{d \lambda^{\prime}}-\frac{d T}{d \lambda}=0$, \&c., analogues aux équations de Dynamique. Mais je n'ai encore rien trouvé sur ce sujet. 